\title{
二茂铁基 1,2,3-三唑化合物的电化学与热性质
}

\author{
徐建伟王绍辉李旸赵海英*李保国边占喜 \\ (内蒙古大学化学化工学院 内蒙古自治区精细有机合成重点实验室 呼和浩特 010021)
}

\begin{abstract}
摘要 合成并表征了含有硝基 $(\mathbf{3 a} \sim \mathbf{3 c})$ 和氨基 $(\mathbf{4 a} \sim \mathbf{4 c})$ 的两系列二茂铁 1,2,3-三唑化合物. 循环伏安实验中代表化合物 $3 \mathbf{b}$ 与 $4 \mathrm{~b}$ 均展示准可逆的单电子氧化还原进程，且相对于 $\mathbf{3 b}$, 化合物 $\mathbf{4 b}$ 的氧化还原电位向阴极方向移动 $17 \mathrm{mV}$ 左右, 说明含有氨基的 $4 \mathrm{~b}$ 更易于氧化. 热性质研究表明, 含有硝基的系列化合物在升温或降温过程中发生了多晶相转变; 而 含有氨基的系列化合物随着烷基链的延长, 出现了晶体到介晶相的转变过程, 且介晶相温度范围可宽达 $90{ }^{\circ} \mathrm{C}$. 这些研 究为此类化合物在功能材料方面的应用提供一定的理论依据.
\end{abstract}

关键词 二茂铁; 1,2,3-三唑; 电化学; 热性质

\section{Electrochemical and Thermal Properties of Ferrocenyl 1,2,3-Triazole}

\author{
$\mathrm{Xu}$, Jianwei Wang, Shaohui $\quad$ Li, Yang Zhao, Haiying* Li, Baoguo $\quad$ Bian, Zhanxi \\ (Inner Mongolia Key Laboratory of Fine Organic Synthesis and College of Chemistry and Chemical Engineering, \\ Inner Mongolia University, Hohhot 010021)
}

\begin{abstract}
Ferrocenyl 1,2,3-triazoles with nitro $(\mathbf{3 a} \sim \mathbf{3 c})$ or amino $(\mathbf{4 a} \sim \mathbf{4 c})$ were synthesized and characterized. The cyclic voltammogram investigations revealed that redox-active ferrocenyl groups of two series of compounds exhibited quasireversible one-electron transfer processes. As compared to compound $\mathbf{3 b}$, the potential for $\mathbf{4 b}$ with amino cathodically shifted $17 \mathrm{mV}$ thereby suggesting easier oxidation by loss of an electron for the latter. Their thermal behaviors indicated that compounds $\mathbf{3 a} \sim$ 3c were all non-mesomorphic, and showed crystal polymorphic phase transitions in the heating and cooling cycles. Whereas 4a $\sim \mathbf{4 c}$ displayed liquid crystal behaviors over $90{ }^{\circ} \mathrm{C}$ mesophase range. The research is supposed to provide some theoretical basis for this kind of compound using in function materials.
\end{abstract}

Keywords ferrocene; 1,2,3-triazole; electrochemistry; thermal behavior

五元杂环化合物 1,2,3-三唑由于具有芳香性, 较高 的极性及化学稳定性, 近年来在化学、材料及药物开发 等领域广泛应用 ${ }^{[1]}$. 其有效的合成方法是基于 Sharpless 及其合作者于 2001 年发现的一价铜催化的有机叠氮与 端炔的 “Click” 反应 ${ }^{[2]}$. 通过该反应可以模块化、方便 高效地构筑 1,2,3-三唑环连接二元及多元功能分子. 当 这些功能分子其中一个单元是二茂铁时, 由于二茂铁具 有较高的热稳定性及优良的电化学性质, 又赋予了该类 化合物新的性能. 目前, 含有二茂铁和三唑环的功能分 子已被合成并应用于多个领域, 如通过三唑环连接的二 茂铁-环糊精二元自组装主客体化合物 ${ }^{[3]}$ 、二茂铁-复合 糖水溶性生物传感 ${ }^{[4]}$ 、二茂铁-碳纳米太阳能电池材
料 ${ }^{[5]}$ 、二茂铁-手性膦配体 ${ }^{[6]}$ 、二茂铁-青霉素类抗生素 等 ${ }^{[7]}$. 这些研究都充分利用了二茂铁卓越的光学、电化 学、磁性及立体结构等特性.

有关二茂铁或三唑化合物热性质方面的研究，文献 上有较多报道 ${ }^{[8,9]}, 1,2,3$-三唑环桥连的盘状或棒状有机 液晶分子均具有较高的热稳定性 ${ }^{[10 \sim 12]}$, 部分单取代和 $1,1^{\prime}$-双取代二茂铁衍生物是性能优良的液晶材料 ${ }^{[13,14]}$. 但是, 将两者相结合, 有关二茂铁取代的三唑化合物热 性质方面的研究非常少见, 我们小组最近通过二茂铁炔 酮与叠氮钠的 1,3-偶极环加成反应合成了系列二茂铁 1,2,3-(NH)-三唑化合物 ${ }^{[15]}$, 研究了其热化学性质, 发现 此类化合物具有液晶性，这是首例二茂铁三唑类金属有

\footnotetext{
*E-mail: hyzhao@imu.edu.cn

Received February 15, 2015; revised March 20, 2015; published online March 24, 2015.
}

Project supported by the National Natural Science Foundation of China (No. 21102068), the Natural Science Foundation of Inner Mongolia (No. 2013MS0207) and the Research Program of Science and Technology at Universities of Inner Mongolia (Nos. NJZZ001, 2014JQ02).

国家自然科学基金(No. 21102068)、内蒙古自治区自然科学基金(No. 2013MS0207)和内蒙古自治区高等学校科学研究(Nos. NJZZ001, 2014JQ02)资助 项目. 
机液晶化合物. 为了开发新的性能优良的二茂铁三唑类 金属有机液晶材料, 本文合成了含有硝基和氨基的两系 列二茂铁三唑化合物(Scheme 1), 通过紫外-可见吸收 光谱(UV-vis)与循环伏安法 (CV)研究该类化合物的光谱 和电化学性质, 通过示差扫描量热分析(DSC)以及热台 偏光显微镜(POM)对化合物的热性质进行研究.

\section{1 结果与讨论}

\section{1 合成与表征}

关于化合物 $3 \mathrm{a} \sim 3 \mathrm{c}$ 的合成, 曾采用二茂铁乙炔与 4-叠氮基-3-硝基苯酚首先在 $\mathrm{Cu}(\mathrm{I})$ 催化下进行 “Click” 反应, 然后再导入长链烷基的方法, 但是由于不带长链 烷基的二茂铁三唑化合物溶解性较差, 致使后续反应无 法进行, 因此改用如 Scheme 1 所示, 将 4-氨基-3-硝基苯 酚先连接上长链烷基, 经叠氮化反应, 得到化合物 $\mathbf{2}$, 然后将 2 与二茂铁乙炔进行 “Click” 反应, 成功得到二 茂铁基三坐化合物 3a $\sim 3 c$. 将化合物 $3 a \sim 3 c$ 中的硝基 用 $\mathrm{Pc} / \mathrm{C}-\mathrm{NaBH}_{4}$ 还原, 得到化合物 $\mathbf{4 a} \sim \mathbf{4 c}$. 化合物 $\mathbf{3} \sim \mathbf{4}$ 的结构通过红外光谱(IR), 核磁共振光谱(NMR)与高分 辨率质谱(HRMS)进行了表征. 在 HRMS 中, 化合物 $\mathbf{3}$ 与 4 分别出现了 $[\mathrm{M}]^{+}$与 $[\mathrm{M}+\mathrm{H}]^{+}$峰, 与计算值一致. 在 化合物 3a $\sim 3 \mathbf{c}$ 的 IR 中, $1350 \mathrm{~cm}^{-1}$ 处的吸收峰归属于硝 基的对称伸缩振动, 还原为化合物 $\mathbf{4 a} \sim \mathbf{4 c}$ 后, 该峰消 失, 而在 $3390 \mathrm{~cm}^{-1}$ 及 $3120 \mathrm{~cm}^{-1}$ 处出现了氨基的特征吸 收. 在 ${ }^{1} \mathrm{H}$ NMR 中, 化合物 $\mathbf{3}$ 与 $\mathbf{4}$ 三唑环上氢原子的化 学位移位于 $\delta 7.66 \sim 7.69$ 处.

图 1 给出了浓度为 $5 \times 10^{-5} \mathrm{~mol} / \mathrm{L}$ 的 $\mathbf{3 a}$ 与 $\mathbf{4 a}$ 在 $\mathrm{CH}_{2} \mathrm{Cl}_{2}$ 中的 UV-vis 光谱, 从图中可知, 化合物 $\mathbf{3 a}$ 与 $\mathbf{4 a}$ 的 UV-vis 光谱是二茂铁与芳环的光谱叠加, 其中 300 $\mathrm{nm}$ 以下的吸收带归属于 $\mathrm{Fe}(\mathrm{d})-\pi *$ 和芳基的 $\pi-\pi^{*}\left(\mathrm{E}_{2}\right.$ 吸收 带)跃迁. 在 3a 中, 由于苯环连接 $\mathrm{NO}_{2}$ 发色团, $\mathrm{B}$ 带吸收 红移至 $333 \mathrm{~nm}$ 附近, 而在硝基还原为氨基后的化合物 4a 中, 该吸收带出现在 $307 \mathrm{~nm}$, 发生了 $25 \mathrm{~nm}$ 左右的蓝 移, 说明硝基发色团取代苯环使共轭体系增大的较显 著, 电子跃迁能级降低. 此外, 从图 1 插图可以看出, 长
波区域放大后, 420 520 nm 处弱而宽的吸收归属于 $\mathrm{Fe}$ 原子的 d-d 跃迁 ${ }^{[16]}$.

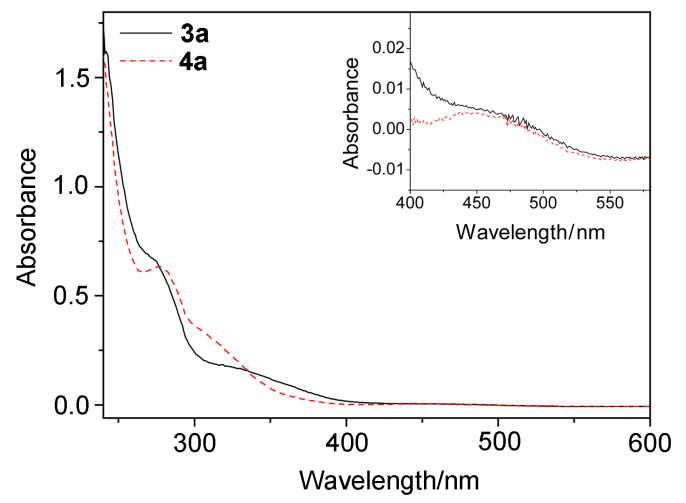

图 1 浓度为 $5.0 \times 10^{-5} \mathrm{~mol} / \mathrm{L}$ 的 $\mathbf{3 a}$ 与 $\mathbf{4 a}$ 在 $\mathrm{CH}_{2} \mathrm{Cl}_{2}$ 中 $\mathrm{UV}$-vis 光谱, 插图是 $400 \sim 580 \mathrm{~nm}$ 处的放大

Figure 1 UV-vis absorption spectra of $\mathbf{3 a}$ and $\mathbf{4 a}$ in $\mathrm{CH}_{2} \mathrm{Cl}_{2}$ at $5.0 \times 10^{-5} \mathrm{~mol} / \mathrm{L}$, insert shows the magnification of $400 \sim 580 \mathrm{~nm}$ absorption bands

\section{2 电化学性质}

以 $0.1 \mathrm{~mol} / \mathrm{L}$ 四丁基六氟磷酸铵 $\left(\mathrm{TBAPF}_{6}\right)$ 作为支持 电解质, 二茂铁作为外标, 通过 CV 研究了代表化合物 $3 \mathbf{b}$ 与 $4 \mathbf{b}$ 浓度为 $1.2 \times 10^{-3} \mathrm{~mol} / \mathrm{L} \mathrm{CH}_{2} \mathrm{Cl}_{2}$ 溶液的电化学性 质，相应的数据列于表 1 . 相对于二茂铁外标 $\mathrm{Fc} / \mathrm{Fc}^{+}$，由 于三唑环的拉电子效应, 化合物 $\mathbf{3 b}$ 与 $\mathbf{3 f}$ 的氧化还原电 位均向阳极发生了移动, 稳定性增加. 其中带有硝基的 $3 \mathbf{b}$, 其氧化还原电位出现在 $E_{1 / 2}=60 \mathrm{mV}$, 而将硝基替 换为氨基后, $\mathbf{4 b}$ 的氧化还原电位向阴极方向移动 $17 \mathrm{mV}$ 左右，说明硝基的拉电子作用进一步加大了二茂铁的氧

表 1 化合物 $\mathbf{3 b}$ 与 $\mathbf{4 b}$ 在 $\mathrm{CH}_{2} \mathrm{Cl}_{2}$ 溶液中的 $\mathrm{CV}$ 数据 $\left(\mathrm{vs} \mathrm{Fc} / \mathrm{Fc}^{+}\right.$) Table $1 \mathrm{CV}$ data of $1.2 \times 10^{-3} \mathrm{~mol} / \mathrm{L}$ solutions of $\mathbf{3 b}$ and $4 \mathbf{b}$ in $\mathrm{CH}_{2} \mathrm{Cl}_{2}$. Potentials are vs. $\mathrm{Fc} / \mathrm{Fc}^{+}$

\begin{tabular}{ccccccc}
\hline Compd. & $E_{\mathrm{pa}}{ }^{a} / \mathrm{mV}$ & $E_{\mathrm{pc}}{ }^{b} / \mathrm{mV}$ & $\Delta E^{c} / \mathrm{mV}$ & $E_{1 / 2}{ }^{d} / \mathrm{mV}$ & $i_{\mathrm{pa}} / \mu \mathrm{A}$ & $i_{\mathrm{pa}} / i_{\mathrm{pc}}$ \\
\hline 3b & 141 & -22 & 163 & 60 & 72.3 & 0.99 \\
4b & 111 & -28 & 139 & 42 & 56.6 & 0.99 \\
\hline
\end{tabular}

${ }^{a}$ Anodic peak potential. ${ }^{b}$ Cathodic peak potential. ${ }^{c} \Delta E=E_{\mathrm{pa}}-E_{\mathrm{pc}} \cdot{ }^{d} E_{1 / 2}=$ $1 / 2\left(E_{\mathrm{pa}}+E_{\mathrm{pc}}\right)$.

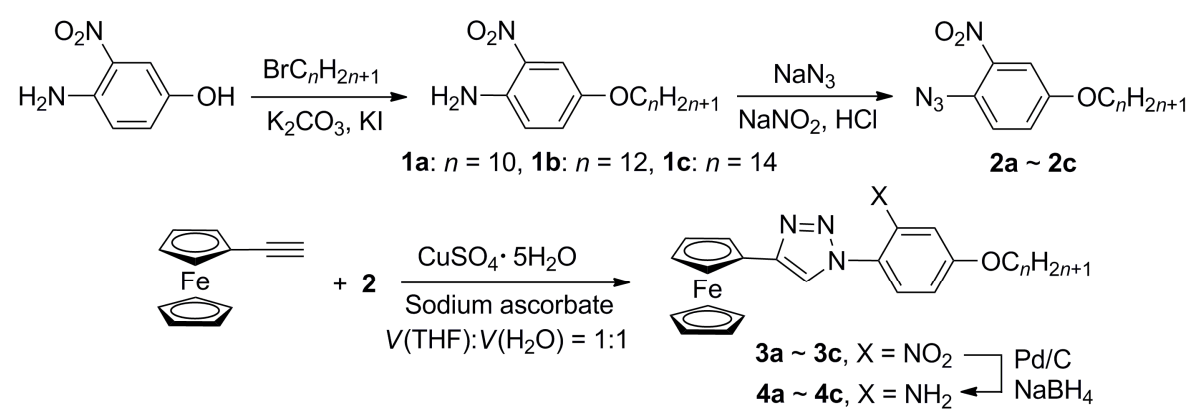

图式 1 化合物 3 4 的合成

Scheme 1 Synthesis of compounds $3 \sim 4$ 
化还原难度. 如表 1 所示, 当扫描速率为 $50 \mathrm{mV} \cdot \mathrm{s}^{-1}$ 时, $i_{\mathrm{pc}} / i_{\mathrm{pa}}$ 接近于 1 , 但是 $\Delta E$ 值在 $140 \sim 160 \mathrm{mV}$ 左右, 理论 上, 当发生电化学可逆的一个电子的氧化还原进程时, $\Delta E=59 \mathrm{mV}$, 说明这是一个准可逆的氧化还原进程 ${ }^{[17]}$.

\section{3 热性质}

通过 DSC 及 POM 对化合物 $3 \sim 4$ 的热性质进行研 究. 有关数据列于表 2 , 代表化合物的 POM 照片见图 2, 化合物 3 4 的 DSC 曲线见支持材料. 发现氮气氛围下, 两系列化合物在 $200{ }^{\circ} \mathrm{C}$ 之前均能稳定存在. 化合物 $3 \mathrm{a} \sim 3 \mathrm{c}$ 在加热过程中出现了从一种晶体到另一种晶体 的多晶相转变. 对于化合物 $\mathbf{3 a}$, 加热时在 $95.8{ }^{\circ} \mathrm{C}$ 及 $122.6{ }^{\circ} \mathrm{C}$ 出现的两个吸热峰分别属于晶体 1 到晶体 2 $\left(\mathrm{C}_{1} \rightarrow \mathrm{C}_{2}\right)$ 及晶体 2 到各向同性液体的相转变过程 $\left(\mathrm{C}_{2} \rightarrow \mathrm{I}\right)$, 且碳链由 10 个碳原子 $\left(122.6{ }^{\circ} \mathrm{C}\right)$ 延长至 12 个碳原子 $\left(127.5{ }^{\circ} \mathrm{C}\right)$ 时, 熔点略有升高, 但是进一步延长至 14 个 碳原子 $\left(109.5{ }^{\circ} \mathrm{C}\right)$ 时, 熔点出现下降趋势. 在升热过程 中, 由于化合物 $3 \mathbf{a}$ 的温度超出了降解温度, 因此未得到 理想的降温及二次升温 DSC 曲线. 化合物 $\mathbf{3 b}$ 在降温时 仅在 $8.4{ }^{\circ} \mathrm{C}$ 出现了玻璃化转变 $T_{\mathrm{g}}$, 二次升温时, $T_{\mathrm{g}}$ 仍然 出现, 随后在 $74.1{ }^{\circ} \mathrm{C}$ 出现了一个产生新晶体的放热峰, 这是因为降温过程中形成的部分玻璃态化合物在二次 升温过程中, 随着分子的流动性增加, 分子重新进行有
序堆积的结果 ${ }^{[18]}$. 与化合物 $\mathbf{3 b}$ 不同的是, DSC 数据显示 $3 \mathrm{c}$ 在降温过程中析出了稳定的晶体, 这从 POM 实验也 得到了证实, 图 2(a) 是化合物 3c 降温到 $43.7{ }^{\circ} \mathrm{C}$ 时析出 的球晶照片.

将化合物 3 中的硝基还原为氨基后，从表 2 可以看 出, 化合物 $4 \mathrm{a}$ 在加热过程中仅出现单一的稳定晶体熔 解过程. 而将长链延长为 12 和 14 个碳原子时, 在加热 或冷却过程 $4 \mathrm{~b}$ 和 $4 \mathrm{c}$ 分别出现了晶体到介晶相的转变. 化合物 $4 \mathrm{~b}$ 在升温过程中, 仍表现为多晶相转变过程, 而 在降温时, 在 $135{ }^{\circ} \mathrm{C}$ 开始出现了介晶相, 持续至 $85{ }^{\circ} \mathrm{C}$. 图 2(b)给出了化合物 $\mathbf{4 b}$ 降温至 $90{ }^{\circ} \mathrm{C}$ 时的花瓣形 $\mathrm{POM}$ 织构. 而化合物 $\mathbf{4 c}$ 在加热过程中就出现了介晶相, 温度 范围从 $84{ }^{\circ} \mathrm{C}$ 至 $177{ }^{\circ} \mathrm{C}$, 介晶相温度范围由 $4 \mathbf{b}$ 的 $50{ }^{\circ} \mathrm{C}$ 增加为 $90{ }^{\circ} \mathrm{C}$ 左右, 清亮点也增加了近 $40{ }^{\circ} \mathrm{C}$. 说明对于 系列化合物 4 , 增加烷基链的长度有助于液晶性的产生 及加宽介晶相温度范围. 此外, 将硝基替换为氨基后有 助于液晶性的产生, 这可能由于氨基形成的分子间氢键 更有利于分子有序排列所致 ${ }^{[11]}$.

\section{2 结论}

合成并表征了含有硝基 $(3 \mathrm{a} \sim 3 \mathrm{c})$ 和氨基 $(4 \mathrm{a} \sim 4 \mathrm{c})$ 的 两系列二茂铁三唑化合物. UV-vis 光谱是二茂铁与芳环

表 2 化合物 $3 \sim \mathbf{4}$ 的相转变温度和晗变

Table 2 Phase transition temperatures and associated enthalpies of compounds $3 \sim \mathbf{4}$

\begin{tabular}{|c|c|c|c|}
\hline \multirow{2}{*}{ Compd. } & \multicolumn{3}{|c|}{ Phase transitions ${ }^{a} /{ }^{\circ} \mathrm{C}\left[\Delta H /\left(\mathrm{kJ}^{\prime} \cdot \mathrm{mol}^{-1}\right)\right]$} \\
\hline & First heating & Second heating & First cooling \\
\hline 3a & $\mathrm{C}_{1} 95.8(4.0) \mathrm{C}_{2} 122.6$ (32.9) I 210 (D) & - & - \\
\hline $\mathbf{3 b}$ & $\mathrm{C}_{1} 123.0 \mathrm{C}_{2} 127.5(40.0)^{b} \mathrm{I}$ & $T_{\mathrm{g}} 10.0 \mathrm{C}_{1} 74.11(-16.8) \mathrm{C}_{2} 140.0(12.7) \mathrm{I}$ & I $8.4 T_{\mathrm{g}}$ \\
\hline $3 c$ & $\mathrm{C}_{1} 53.5(10.4) \mathrm{C}_{2} 102.5 \mathrm{C}_{3} 109.5(52.3)^{b} \mathrm{I}$ & $\mathrm{C}_{1} 14.5(-2.9) \mathrm{C}_{2} 106.6(27.8) \mathrm{I}$ & I $19.5(-9.2) \mathrm{C}$ \\
\hline $4 a$ & C $103.3(51.4) \mathrm{I}$ & C $65.7(3.4) \mathrm{I}$ & - \\
\hline $4 b$ & $\mathrm{C}_{1} 103.9(9.0) \mathrm{C}_{2} 149.7(27.2) \mathrm{I}$ & $\begin{array}{l}\mathrm{C}_{1} 75.3(-4.0) \mathrm{C}_{2} 100.4(2.2) \mathrm{C}_{3} 121.4(-4.4) \\
\mathrm{C}_{4} 153.5(6.7) \mathrm{I}\end{array}$ & $\begin{array}{l}\text { I } 135.0(-5.9) \\
\text { M } 85.2(-12.6)\end{array}$ \\
\hline $4 c$ & C $83.7(39.8)$ M $177.4(-17.0) \mathrm{I}$ & $T_{\mathrm{g}} 1.7 \mathrm{C} 74.8(6.3) \mathrm{I}$ & $\mathrm{I}-1.3 T_{\mathrm{g}}$ \\
\hline
\end{tabular}

${ }^{a} \mathrm{C}$ : crystal; M: mesophase; I: isotropic; $T_{\mathrm{g}}$ : glass transition; D: degradation. ${ }^{b}$ Two peaks joint enthalpy.

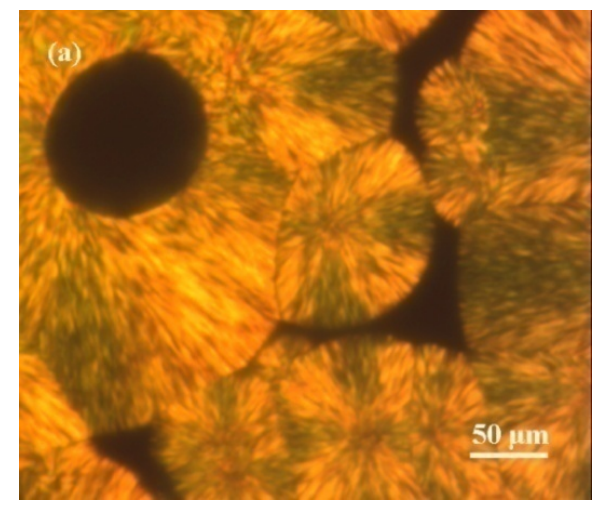

图 2 降温过程中化合物 $\mathbf{3 c}$ 在 $43.7^{\circ} \mathrm{C}$

Figure 2 POM images of $3 \mathbf{c}$ at $43.7{ }^{\circ} \mathrm{C}$

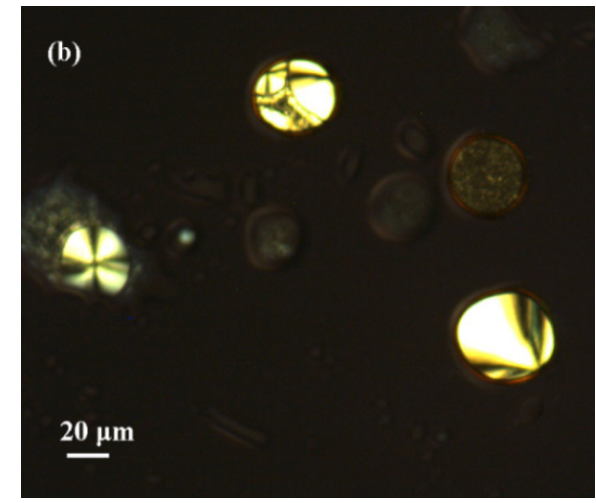

(a)及 $4 \mathbf{b}$ 在 $90{ }^{\circ} \mathrm{C}$ (b) 的 POM 照片 (a) and $\mathbf{4 b}$ at $90{ }^{\circ} \mathrm{C}$ (b) on the cooling 
的光谱叠加, 由于化合物 3 中苯环上连接 $\mathrm{NO}_{2}$ 发色团, 其 B 带吸收较化合物 4 发生了 $25 \mathrm{~nm}$ 左右的红移. 这两 系列化合物均展示较好的电化学性质, 且相对于含有硝 基的系列化合物，含有氨基的 $\mathbf{4 a} \sim \mathbf{4 c}$ 更易于氧化. 热性 质研究表明, 含有硝基的系列化无液晶性, 在升温或降 温过程中发生了多晶相转变; 而含有氨基的系列化合由 于分子间氢键的作用, 出现了晶体到介晶相的转变过 程, 且介晶相温度范围较宽. 这些研究为此类化合物在 功能材料方面的应用提供一定的借鉴意义.

\section{3 实验部分}

\section{1 仪器与试剂}

化合物 1 与 2 的制备见辅助材料. NEXUS-670 傅里 叶变换红外光谱仪, $\mathrm{KBr}$ 压片; Avance 500 Bruker (500 $\mathrm{MHz}$ ) 核磁共振仪, 以 $\mathrm{TMS}$ 为内标; Analytikjena SPECORD.50UV-vis 光谱仪; Edinburgh Instruments FLS920 苂光光谱仪; IonSpec 710TFTICR/MS 高分辨质 谱仪(ESI 源); DSC 测试使用 TA Q2000 热分析仪, 氮气 氛围, 加热速率 $10{ }^{\circ} \mathrm{C} \cdot \mathrm{min}^{-1}$; 莱卡 DMLP 偏光显微镜, THMS600 冷热台, 加热冷却速率 $10{ }^{\circ} \mathrm{C} \cdot \mathrm{min}^{-1}$; $\mathrm{AU}$ TOLAB PGSTAT302 电化学分析仪, 在 $298 \mathrm{~K}$ 下, 样品 溶于干燥的 $\mathrm{CH}_{2} \mathrm{Cl}_{2}$ 溶液, 加入 $0.1 \mathrm{~mol} \cdot \mathrm{L}^{-1}$ 的 $\mathrm{TBAPF}_{6}$ 作为支持电解质, 用三电极方法, 玻碳电极为工作电极, $\mathrm{Pt}$ 为对电极, $\mathrm{Ag} / \mathrm{AgCl}$ 为参比电极, $\mathrm{Fc} / \mathrm{Fc}^{+}$作为外标, 其 电势为 $+0.564 \mathrm{~V}$. 所用试剂均为市售分析纯试剂, 溶剂 的干燥按常规方法进行.

\section{2 实验方法}

\section{2 .1 化合物 $\mathbf{3 a} \sim \mathbf{3} \mathbf{c}$ 的合成}

在 $100 \mathrm{~mL}$ 圆底烧瓶中, 加入化合物 $2(0.50 \mathrm{mmol})$, 二茂铁乙㶧 $(0.11 \mathrm{~g}, 0.55 \mathrm{mmol})$, 四氢呋喃 $10 \mathrm{~mL}$, 室温 下将 $\mathrm{CuSO}_{4} \cdot 5 \mathrm{H}_{2} \mathrm{O}(0.13 \mathrm{~g}, 0.5 \mathrm{mmol})$ 溶于 $5 \mathrm{~mL}$ 水中, 缓 慢滴入上述反应液. 滴毕, 再缓慢滴入抗坏血酸钠 $(0.29$ $\mathrm{g}, 1.5 \mathrm{mmol}$ )溶于 $5 \mathrm{~mL}$ 的水溶液, 此时反应液颜色由黄 变红. 室温下搅拌 $4 \mathrm{~h}$, 停止反应. 减压蒸除溶剂, 剩余 物用硅胶柱层析, 石油醚-乙酸乙酯(4: $1, V: V)$ 淋洗, 收集第 $\mathrm{I}$ 带黄色产物, 浓缩, 用无水甲醇- $\mathrm{CH}_{2} \mathrm{Cl}_{2}$ 重结晶 得黄色粉末 3 .

1-(2-硝基-4-癸烷氧基苯基)-4-二茂铁基- $1 H-1,2,3$ 三唑(3a): 产率 90\%. m.p. 122 123 ${ }^{\circ} \mathrm{C}$; UV-Vis $\left(\mathrm{CHCl}_{3}\right)$

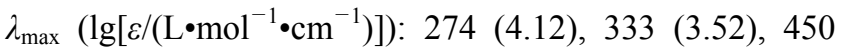
(2.25) nm; Fluorescence $\left(\mathrm{CH}_{2} \mathrm{Cl}_{2}, \lambda_{\mathrm{ex}}=275 \mathrm{~nm}\right) \lambda_{\mathrm{em}}: 336$ $\mathrm{nm} ;{ }^{1} \mathrm{H} \mathrm{NMR}\left(\mathrm{CDCl}_{3}, 500 \mathrm{MHz}\right) \delta: 7.66(\mathrm{~s}, 1 \mathrm{H}$, triazole), 7.58 (s, 1H, phenyl-H), 7.51 (s, 1H, phenyl-H), $7.25(\mathrm{~s}, 1 \mathrm{H}$, phenyl-H), 4.94 (s, 2H, FcH), 4.49 (s, 2H, FcH), 4.26 (s, $5 \mathrm{H}, \mathrm{FcH}), 4.05\left(\mathrm{t}, J=6.5 \mathrm{~Hz}, 2 \mathrm{H}, \mathrm{OCH}_{2}\right), 1.88 \sim 1.83(\mathrm{~m}$, $\left.2 \mathrm{H}, \mathrm{CH}_{2}\right), 1.51 \sim 1.46\left(\mathrm{~m}, 2 \mathrm{H}, \mathrm{CH}_{2}\right), 1.36 \sim 1.26(\mathrm{~m}, 12 \mathrm{H}$, $\mathrm{CH}_{2}$ ), 0.89 (t, $\left.J=6.5 \mathrm{~Hz}, 3 \mathrm{H}, \mathrm{CH}_{3}\right)$; IR $(\mathrm{KBr}) v: 3137$, 3079, 2920, 2850, 1558, 1515, 1345, 1256, 1041, 839, 810, $512 \mathrm{~cm}^{-1}$. HRMS calcd for $\mathrm{C}_{28} \mathrm{H}_{34} \mathrm{FeN}_{4} \mathrm{O}_{3} 530.1980$, found 530.1978

1-(2-硝基-4-十二烷氧基苯基)-4-二茂铁基- $1 H$ 1,2,3-三唑(3b): 产率 93\%. m.p. $127 \sim 128{ }^{\circ} \mathrm{C}$; UV-Vis

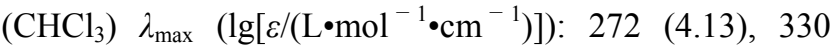
(3.50), 452 (2.25) nm; Fluorescence $\left(\mathrm{CH}_{2} \mathrm{Cl}_{2}, \lambda_{\mathrm{ex}}=275\right.$ nm) $\lambda_{\mathrm{em}}: 336 \mathrm{~nm} ;{ }^{1} \mathrm{H}$ NMR $\left(\mathrm{CDCl}_{3}, 500 \mathrm{MHz}\right) \delta: 7.68(\mathrm{~s}$, $1 \mathrm{H}$, triazole), $7.58(\mathrm{~s}, 1 \mathrm{H}$, phenyl-H), $7.54(\mathrm{~d}, J=8.5 \mathrm{~Hz}$, $1 \mathrm{H}$, phenyl-H), 7.25 (d, $J=8.5 \mathrm{~Hz}, 1 \mathrm{H}$, phenyl-H), 4.81 (s, 2H, FcH), 4.35 (s, 2H, FcH), 4.14 (s, 5H, FcH), 4.09 (t, $J=$ $\left.6.5 \mathrm{~Hz}, 2 \mathrm{H}, \mathrm{OCH}_{2}\right), 1.88 \sim 1.83\left(\mathrm{~m}, 2 \mathrm{H}, \mathrm{CH}_{2}\right), 1.50 \sim 1.46$ $\left(\mathrm{m}, 2 \mathrm{H}, \mathrm{CH}_{2}\right), 1.39 \sim 1.26\left(\mathrm{~m}, 16 \mathrm{H}, \mathrm{CH}_{2}\right), 0.89(\mathrm{t}, J=6.5$ $\left.\mathrm{Hz}, 3 \mathrm{H}, \mathrm{CH}_{3}\right) ;{ }^{13} \mathrm{C}$ NMR $\left(\mathrm{CDCl}_{3}, 125 \mathrm{MHz}\right) \delta: 160.33$, $147.35,145.19,129.32,122.98,120.63,119.73,110.92$, $69.86,69.46,68.96,66.84,31.92,29.65,29.63,29.58$, $29.53,29.35,29.29,28.86,25.87,22.69,14.13$; IR (KBr) $v: 3137,3080,2921,2851,1557,1518,1467,1352,1259$, $1104,1041,841,810,512 \mathrm{~cm}^{-1}$. HRMS calcd for $\mathrm{C}_{30} \mathrm{H}_{38^{-}}$ $\mathrm{FeN}_{4} \mathrm{O}_{3}$ 558.2293, found 558.2285.

1-(2-硝基-4-十四烷氧基苯基)-4-二茂铁基- $1 H$ 1,2,3-三唑(3c): 产率 91\%. m.p. $106 \sim 107{ }^{\circ} \mathrm{C}$; UV-Vis

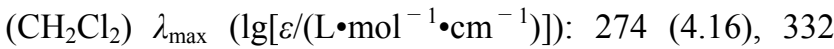
(3.51), 450 (2.25) nm; Fluorescence $\left(\mathrm{CH}_{2} \mathrm{Cl}_{2}, \lambda_{\mathrm{ex}}=275\right.$ nm) $\lambda_{\mathrm{em}}: 336 \mathrm{~nm} ;{ }^{1} \mathrm{H}$ NMR $\left(\mathrm{CDCl}_{3}, 500 \mathrm{MHz}\right) \delta: 7.68(\mathrm{~s}$, $1 \mathrm{H}$, triazole), 7.58 (s, $1 \mathrm{H}$, phenyl-H), $7.54(\mathrm{~d}, J=8.5 \mathrm{~Hz}$, $1 \mathrm{H}$, phenyl-H), 7.24 (br s, 1H, phenyl-H), $4.82(\mathrm{~s}, 2 \mathrm{H}$, $\mathrm{FcH}), 4.36$ (s, 2H, FcH), 4.14 (s, 5H, FcH), 4.09 (t, $J=6.5$ $\left.\mathrm{Hz}, 2 \mathrm{H}, \mathrm{OCH}_{2}\right), 1.88 \sim 1.83\left(\mathrm{~m}, 2 \mathrm{H}, \mathrm{CH}_{2}\right), 1.52 \sim 1.46(\mathrm{~m}$, $\left.2 \mathrm{H}, \mathrm{CH}_{2}\right), 1.39 \sim 1.26\left(\mathrm{~m}, 20 \mathrm{H}, \mathrm{CH}_{2}\right), 0.89$ (t, $J=6.5 \mathrm{~Hz}$, $3 \mathrm{H}, \mathrm{CH}_{3}$ ); IR (KBr) v: 3110, 2920, 2851, 1558, 1519, $1401,1354,1258,1104,1043,841,812,512 \mathrm{~cm}^{-1}$. HRMS calcd for $\mathrm{C}_{32} \mathrm{H}_{42} \mathrm{FeN}_{4} \mathrm{O}_{3}$ 586.2606, found 586.2606.

\section{2 .2 化合物 $\mathbf{4 a} \sim \mathbf{4 c}$ 的合成}

在 $50 \mathrm{~mL}$ 圆底烧瓶中, 加入化合物 $3(0.5 \mathrm{mmol})$, 然 后加入 $30 \mathrm{~mL} \mathrm{CH} \mathrm{Cl}_{2}$-无水甲醇 $(V: V=1: 1)$ 混合溶液 使之溶解，室温摚拌下加入经无水甲醇洗涤过的钯碳 $(5 \%, 100 \mathrm{mg})$. 在 $10 \mathrm{~min}$ 内分三次向体系中加入 $\mathrm{NaBH}_{4}$ (60 mg, $1.5 \mathrm{mmol})$, 此时有气泡产生. 室温下继续摚拌 $0.5 \mathrm{~h}$ ，停止反应. 抽滤除去钯碳，将滤液用蒸馏水洗涤 二次除去未反应的 $\mathrm{NaBH}_{4}$, 无水 $\mathrm{MgSO}_{4}$ 干燥、过滤、减 压蒸除溶剂，剩余物用硅胶柱层析，石油醚-乙酸乙酯 $(V: V=2: 1)$ 洗脱. 收集第 II 带黄色产物, 浓缩, 用无 
水甲醇- $\mathrm{CH}_{2} \mathrm{Cl}_{2}$ 重结晶得到黄色粉末 4 .

2-(4-二茂铁基-1H-1,2,3-三唑基)-5-癸烷氧基苯胺 (4a): 产率 89\%. m.p. 103 104 ${ }^{\circ} \mathrm{C}$; UV-Vis $\left(\mathrm{CHCl}_{3}\right) \lambda_{\max }$ $\left(\lg \left[\varepsilon /\left(\mathrm{L} \cdot \mathrm{mol}^{-1} \cdot \mathrm{cm}^{-1}\right)\right]\right): 277$ (4.10), 307 (3.82), 450 (2.15) $\mathrm{nm}$; Fluorescence $\left(\mathrm{CH}_{2} \mathrm{Cl}_{2}, \lambda_{\mathrm{ex}}=275 \mathrm{~nm}\right) \lambda_{\mathrm{em}}: 336 \mathrm{~nm} ;{ }^{1} \mathrm{H}$ NMR $\left(\mathrm{CDCl}_{3}, 500 \mathrm{MHz}\right) \delta: 7.69(\mathrm{~s}, 1 \mathrm{H}$, triazole), $7.14(\mathrm{~s}$, 1H, phenyl-H), 6.38 (d, $J=8.5 \mathrm{~Hz}, 2 \mathrm{H}$, phenyl-H), 4.78 (s, 2H, FcH), 4.51 (br s, 2H, $\mathrm{NH}_{2}$ ), 4.33 (s, 2H, FcH), 4.12 (s, $5 \mathrm{H}, \mathrm{FcH}), 3.96\left(\mathrm{t}, J=6.5 \mathrm{~Hz}, 2 \mathrm{H}, \mathrm{OCH}_{2}\right), 1.82 \sim 1.76(\mathrm{~m}$, $\left.2 \mathrm{H}, \mathrm{CH}_{2}\right), 1.49 \sim 1.43\left(\mathrm{~m}, 2 \mathrm{H}, \mathrm{CH}_{2}\right), 1.35 \sim 1.28(\mathrm{~m}, 12 \mathrm{H}$, $\left.\mathrm{CH}_{2}\right), 0.89\left(\mathrm{t}, J=6.5 \mathrm{~Hz}, 3 \mathrm{H}, \mathrm{CH}_{3}\right) ;{ }^{13} \mathrm{C} \mathrm{NMR}\left(\mathrm{CDCl}_{3}, 125\right.$ MHz) $\delta: 160.44,146.59,142.18,125.36,119.65,116.99$, $104.86,102.60$, 69.76, 68.91, 68.26, 66.87, 31.91, 29.57, 29.40, 29.33, 29.20, 26.02, 22.69, 14.14; IR (KBr) v: 3393, 3124, 2917, 2849, 1626, 1520, 1397, 1304, 1206, 1084, $820,516 \mathrm{~cm}^{-1}$. HRMS calcd for $\mathrm{C}_{28} \mathrm{H}_{37} \mathrm{FeN}_{4} \mathrm{O}[\mathrm{M}+\mathrm{H}]^{+}$ 501.2313, found 501.2317.

\section{2-(4-二茂铁基-1H-1,2,3-三唑基)-5-十二烷氧基苯胺} (4b): 产率 92\%. m.p. 149 $150{ }^{\circ} \mathrm{C}$; UV-Vis $\left(\mathrm{CHCl}_{3}\right) \lambda_{\max }$

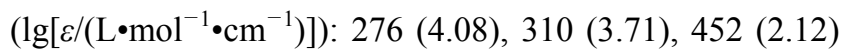
$\mathrm{nm}$; Fluorescence $\left(\mathrm{CH}_{2} \mathrm{Cl}_{2}, \lambda_{\mathrm{ex}}=275 \mathrm{~nm}\right) \lambda_{\mathrm{em}}: 336 \mathrm{~nm} ;{ }^{1} \mathrm{H}$ NMR $\left(\mathrm{CDCl}_{3}, 500 \mathrm{MHz}\right) \delta: 7.69(\mathrm{~s}, 1 \mathrm{H}$, triazole), 7.15 7.13 (m, 1H, phenyl-H), $6.39 \sim 6.37(\mathrm{~m}, J=8.5 \mathrm{~Hz}, 2 \mathrm{H}$, phenyl-H), 4.76 (s, 2H, FcH), 4.54 (s, 2H, $\left.\mathrm{NH}_{2}\right), 4.33$ (s, $2 \mathrm{H}, \mathrm{FcH}), 4.11(\mathrm{~s}, 5 \mathrm{H}, \mathrm{FcH}), 3.95(\mathrm{t}, J=6.5 \mathrm{~Hz}, 2 \mathrm{H}$, $\left.\mathrm{OCH}_{2}\right), 1.80 \sim 1.76\left(\mathrm{~m}, 2 \mathrm{H}, \mathrm{CH}_{2}\right), 1.49 \sim 1.44(\mathrm{~m}, 2 \mathrm{H}$, $\left.\mathrm{CH}_{2}\right), 1.35 \sim 1.28\left(\mathrm{~m}, 16 \mathrm{H}, \mathrm{CH}_{2}\right), 0.89(\mathrm{t}, J=6.5 \mathrm{~Hz}, 3 \mathrm{H}$, $\mathrm{CH}_{3}$ ); IR (KBr) v: 3395, 3121, 2915, 2845, 1621, 1519, 1303, 1206, 1043, 819, $509 \mathrm{~cm}^{-1}$. HRMS calcd for $\mathrm{C}_{30} \mathrm{H}_{41} \mathrm{FeN}_{4} \mathrm{O}[\mathrm{M}+\mathrm{H}]^{+}$529.2630, found 529.2621.

2-(4-二茂铁基-1H-1,2,3-三唑基)-5-十四烷氧基苯胺 (4c): 产率 90\%. m.p. $83 \sim 84{ }^{\circ} \mathrm{C}$; UV-Vis $\left(\mathrm{CHCl}_{3}\right) \lambda_{\max }$

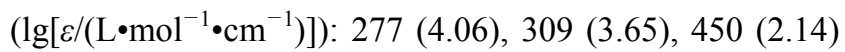
$\mathrm{nm}$; Fluorescence $\left(\mathrm{CH}_{2} \mathrm{Cl}_{2}, \lambda_{\mathrm{ex}}=275 \mathrm{~nm}\right) \lambda_{\mathrm{em}}: 336 \mathrm{~nm} ;{ }^{1} \mathrm{H}$ NMR $\left(\mathrm{CDCl}_{3}, 500 \mathrm{MHz}\right) \delta: 7.68(\mathrm{~s}, 1 \mathrm{H}$, triazole $), 7.14(\mathrm{~d}$, $J=8.0 \mathrm{~Hz}, 1 \mathrm{H}$, phenyl-H), 6.37 (d, $J=8$. Hz, 2H, phenyl-H), 4.79 (s, 2H, FcH), 4.67 (br s, 2H, NH $), 4.35$ (s, $2 \mathrm{H}, \mathrm{FcH}), 4.13(\mathrm{~s}, 5 \mathrm{H}, \mathrm{FcH}), 3.94(\mathrm{t}, J=6.5 \mathrm{~Hz}, 2 \mathrm{H}$, $\left.\mathrm{OCH}_{2}\right), 1.80 \sim 1.76\left(\mathrm{~m}, 2 \mathrm{H}, \mathrm{CH}_{2}\right), 1.46 \sim 1.42(\mathrm{~m}, 2 \mathrm{H}$, $\left.\mathrm{CH}_{2}\right), 1.35 \sim 1.28\left(\mathrm{~m}, 20 \mathrm{H}, \mathrm{CH}_{2}\right), 0.89(\mathrm{t}, J=6.5 \mathrm{~Hz}, 3 \mathrm{H}$,
$\left.\mathrm{CH}_{3}\right) ;{ }^{13} \mathrm{C} \mathrm{NMR}\left(\mathrm{CDCl}_{3}, 125 \mathrm{MHz}\right) \delta: 160.44,146.66$, $142.19,125.39,119.81,116.94,104.88,102.60,70.84$, $69.84,68.27,67.55,31.95,29.71,29.63,29.41,29.38$, 29.21, 26.04, 22.71, 14.15; IR (KBr) v: 3390, 3120, 2919, 2849, 1616, 1518, 1278, 1210, 1044, 816, $481 \mathrm{~cm}^{-1}$. HRMS calcd for $\mathrm{C}_{32} \mathrm{H}_{45} \mathrm{FeN}_{4} \mathrm{O}[\mathrm{M}+\mathrm{H}]^{+}$557.2943, found 557.2943 .

辅助材料(Supporting Information) 化合物 $\mathbf{1} \sim \mathbf{2}$ 的合 成与表征及化合物 3 4 的 NMR 与 HRMS 谱图, DSC 曲 线. 这些材料可以免费从本刊网站(http://sioc-journal. $\mathrm{cn} /$ )上下载.

\section{References}

[1] Ganesh, V.; Sudhir, V. S.; Kundu, T.; Chandrasekaran, S. Chem. Asian J. 2011, 6, 2670.

[2] Kolb, H. C.; Finn, M. G.; Sharpless, K. B. Angew. Chem. 2001, 113, 2056.

[3] Diallo, A. K.; Menuel, S.; Monflier, E.; Ruiz, J.; Astruc, D. Tetrahedron Lett. 2010, 51, 4617.

[4] Casas-Solvas, J. M.; Vargas-Berenguel, A.; Capitán-Vallvey, L. F.; Santoyo-González, F. Org. Lett. 2004, 6, 3687.

[5] Zhao, H. Y.; Zhu, Y. Z.; Chen, C.; He, L.; Zheng, J. Y. Carbon 2012, 50, 4894.

[6] Fukuzawa, S.-I.; Oki, H.; Hosaka, M.; Sugasawa, J.; Kikuchi, S. Org. Lett. 2007, 9, 5557.

[7] Martic, S.; Labib, M.; Shipman, P. O.; Kraatz, H.-B. Dalton Trans. 2011, 40, 7264.

[8] Kadkin, O. N.; Galyametdinov, Y. G. Russ. Chem. Rev. 2012, 81, 675.

[9] Cho, B.-K.; Kim, S. H. Soft Matter 2014, 10, 553.

[10] Yeap, G. Y.; Balamurugan, S.; Srinivasan, M. V.; Kannan, P. New J. Chem. 2013, 37, 1906.

[11] Ryu, M. H.; Choi, J. W.; Kim, H. J.; Park, N.; Cho, B. K. Angew. Chem., Int. Ed. 2011, 50, 5737.

[12] Yu, W. H.; Chen, C.; Hu, P.; Wang, B. Q.; Redshaw, C.; Zhao, K. Q. RSC Adv. 2013, 3, 14099.

[13] Amer, W. A.; Wang, L.; Yu, H.; Amin, A. M.; Wang, Y. J. Inorg. Organomet. Polym. 2012, 22, 1229.

[14] Massiot, P.; Impéror-Clerc, M.; Veber, M.; Deschenaux, R. Chem. Mater. 2005, 17, 1946.

[15] Zhao, H. Y.; Guo, L.; Chen, S. F.; Bian, Z. X. J. Mol. Struct. 2013, 1054,164

[16] Zhao, H. Y.; Zhu, X. Y.; Wang, D.; Chen, S. F.; Bian, Z. X. Aust. J. Chem. 2015, 68, 1035.

[17] Muller, T. J.; Conradie, J.; Erasmus, E. Polyhedron 2012, 33, 257.

[18] Wang, D.; Zhu, X. Y.; Zhao, H. Y.; Bian, Z. X. Chin. J. Org. Chem. 2015, 35, 1131 (in Chinese).

(王栋, 朱学友, 赵海英, 边占喜, 有机化学, 2015, 35, 1131.) 